\title{
A NOTE ON THE POWER OF LEAST SQUARES TESTS FOR A UNIT ROOT
}

\author{
Kenneth D. WEST * \\ Princeton University, Princeton, NJ 08544, USA
}

Received 29 April 1987

Two least squares tests for a unit autoregressive root are inconsistent if the process being studied is stationary around a time trend, and a time trend is not included as a regressor.

\section{Introduction}

Recent research in macroeconomics has emphasized the possibility that there are unit autoregressive roots in macroeconomic time series [e.g., Nelson and Plosser (1982)]. This has led to increased interest in tests for the presence of such roots. Despite the development of some relatively general and powerful procedures [e.g., Bhargava (1986) and Phillips and Perron (1986)], much research uses the simple ordinary least squares tests suggested in the pioneering research of Fuller (1976) and Dickey and Fuller (1979). Some applications use versions of these tests that do not include a time trend as a regressor [e.g., Kleidon (1986) and Mattey and Meese (1986)]. The purpose of this note is to point out that these versions are inconsistent if the series is stationary around a time trend, an alternative that often [e.g., Kleidon (1986) and Mattey and Meese (1986)] is the relevant one. Indeed, the asymptotic distribution of the test statistics is more concentrated around zero under this alternative than under the null.

\section{Model and tests}

The researcher assumes that the covariance stationary first difference of a series $y_{t}$ follows a finite-order autoregression order $k-1(k \geqslant 1)$,

$\Delta y_{t}=m+\phi_{1} \Delta y_{t-1}+\cdots+\phi_{k-1} \Delta y_{t-k+1}+e_{t}$,

where $e_{t}$ is serially uncorrelated and the roots of $1-\phi_{1} z-\ldots \phi_{k} z^{k}$ are outside the unit circle. One way to test for the hypothesized unit root is to rewrite (1) as

$y_{t}=m+\phi_{1} \Delta y_{t-1}+\cdots+\phi_{k-1} \Delta y_{t-k+1}+\gamma y_{t-1}+e_{t}$.

Let $\hat{\gamma}$ be the OLS estimate of $\gamma$. One can compare either $T(\hat{\gamma}-1)$, or the $t$-statistic for $\mathrm{H}_{0}: \gamma=1$, to the values in the tables in Fuller (1976) to test the null. One can also include a time trend as a

* This research was supported by the National Science Foundation. This paper was first written while the author was a National Fellow at the Hoover Institution. 
regressor in (2), and use some other tables in Fuller (1976). In either case, values of $T(\hat{\gamma}-1)$, or of the $t$-statistic, that are far from zero call for rejection of the null.

It seems intuitively obvious that one should include the time trend if the alternative is that $y_{t}$ is stationary around a time trend. This clearly is the intention of Dickey and Fuller (1979) and Fuller (1976), and it is often done by applied researchers [e.g., Nelson and Plosser (1982)]. Sometimes, however, the time trend is omitted even when trend stationarity is the relevant alternative. In tests for a unit root in aggregate stock prices and dividends, for example, the term is omitted in some (but not all) of Kleidon's (1986) tests and in all of Mattey and Meese's (1986) tests; Shiller (1981), in a paper cited by Mattey and Meese (1986) and, especially, by Kleidon (1986), emphasizes that trend stationarity is the alternative of interest. Kleidon (1986, table 6) finds considerably less evidence against the null of a unit root when the time trend is omitted, and indicates in his footnote 29 that this is unsurprising if the series with a unit root has a non-zero drift. Theorem 1 establishes that this is also to be expected if the trend stationary alternative is correct.

\section{Theorem 1. Suppose that}

$y_{t}=m+\alpha_{1} y_{t-1}+\cdots+\alpha_{k} y_{t-k}+\delta t+e_{t}$,

where $k \geqslant 1 ; e_{t}$ is zero mean and serially uncorrelated; the roots of $1-\alpha_{1} z-\ldots \alpha_{k} z^{k}$ are outside the unit circle; $\Delta y_{1}$ is stationary to fourth order and ergodic; $\Delta y_{t}$ has absolutely summable fourth cumulants; $y_{0}, \ldots, y_{-k+1}$ are fixed; and, finally, $\delta \neq 0$. Rewrite (3) as

$y_{t}=m+\phi_{1} \Delta y_{t-1}+\cdots+\phi_{k-1} \Delta y_{t-k+1}+\gamma y_{t-1}+\delta t+e_{t}$,

with $\gamma \equiv \sum_{1}^{k} \alpha_{i},|\gamma|<1$ [Fuller (1976)], and $\phi_{j} \equiv-\sum_{i=j+1}^{k} \alpha_{i}$. Let $\gamma$ be estimated by ordinary least squares by regressing $y_{t}$ on $X_{1 t}^{\prime} \equiv\left(1, \Delta y_{t-1}, \ldots, \Delta y_{t-k+1}\right)$ and $y_{t-1}$. Let $T$ be the sample size. Then $T(\hat{\gamma}-1)$ and the usual $t$-statistic for $\mathrm{H}_{0}: \gamma=1$ each converge in probability to zero.

Proof. Let $X_{1}$ be the $T \times k$ matrix whose $t$ th row is $X_{1 r}^{\prime}$. Define $M_{1}=I-X_{1}\left(X_{1}^{\prime} X_{1}\right)^{-1} X_{1}^{\prime}$. For any scalar random variable $z_{t}$, let $\left[z_{l}\right]$ be the $T \times 1$ vector with $z_{t}$ as its $t$ th element. We have

$\hat{\gamma}=\left\{\left[y_{t-1}\right]^{\prime} M_{1}\left[y_{t-1}\right]\right\}^{-1}\left\{\left[y_{t-1}\right]^{\prime} M_{1}\left[y_{t}\right]\right\}$

$\Rightarrow T^{3 / 2}(\hat{\gamma}-1)=\left\{T^{-3}\left[y_{t-1}\right]^{\prime} M_{1}\left[y_{t-1}\right]\right\}^{-1}\left\{T^{-3 / 2}\left[y_{t-1}\right]^{\prime} M_{1}\left[\Delta y_{t}\right]\right\}$.

It will be shown that $T^{3 / 2}(\hat{\gamma}-1) \stackrel{\mathrm{p}}{\rightarrow} 0$ by showing that (i) $T^{-3}\left[y_{t-1}\right]^{\prime} M_{1}\left[y_{t-1}\right]$ converges in probability to a non-zero constant, and (ii) $T^{-3 / 2}\left[y_{i-1}\right]^{\prime} M_{1}\left[\Delta y_{l}\right] \stackrel{\mathrm{P}}{\rightarrow} 0$. This will establish that $T(\hat{\gamma}-1) \stackrel{p}{\rightarrow} 0$. Then the fact that $T^{3 / 2}(\hat{\gamma}-1) \stackrel{n}{\rightarrow} 0$ will be used to show that (iii) the $t$-statistic for $\mathrm{H}_{0}: \gamma=1$ converges in probability to zero.

(i) By the definition of $M_{1}, \quad\left\{T^{-3}\left[y_{t-1}\right]^{\prime} M_{1}\left\lfloor y_{t-1}\right\rfloor\right\}=T^{-3}\left[y_{t-1}\right]^{\prime}\left\lfloor y_{t-1}\right\rfloor+\left\{T^{-2}\left[y_{t-1}\right]^{\prime} X_{1}\right\}$ $\left\{T^{-1} X_{1}^{\prime} X_{1}\right\}^{-1}\left\{T^{-2} X_{1}^{\prime}\left[y_{t-1}\right]\right\}$. Let $\mu=\mathrm{E} \Delta y_{t}=\delta /\left(1-\alpha_{1}-\cdots-\alpha_{k}\right), \mu \neq 0$, since $\delta \neq 0$ by hypothesis. Recall that a stationary finite-order autoregressive process has an absolutely summable autocovariance function. By Lemma 2.2 in West (1987), then, $T^{-3}\left[y_{t-1}\right]^{\prime}\left[y_{t-1}\right] \stackrel{\text { p }}{\rightarrow}(1 / 3) \mu^{2}$. By Lemma A.3 in West (1987), $\left\{T^{-2}\left[y_{t-1}\right]^{\prime} X_{1}\right\} \stackrel{\mathrm{p}}{\rightarrow}(1 / 2) \mu \mathrm{E} X_{1 i}^{\prime}$. It follows that

$$
\begin{aligned}
\mathrm{p} \lim T^{-3}\left\{\left[y_{t-1}\right]^{\prime} M_{1}\left[y_{t-1}\right]\right\} & =(1 / 3) \mu^{2}-(1 / 2) \mu \mathrm{E} X_{1 t}^{\prime}\left(\mathrm{E} X_{1 t} X_{1 t}^{\prime}\right)^{-1}(1 / 2) \mu \mathrm{E} X_{1 t} \\
& =(1 / 3) \mu^{2}-(1 / 4) \mu^{2} \neq 0 .
\end{aligned}
$$


The second equality follows since $\mathrm{E} X_{1 t}^{\prime}\left(\mathrm{E} X_{1 t} X_{1 t}^{\prime}\right)^{-1} \mathrm{E} X_{1 t}=1$ : let [1] be a $T \times 1$ vector of ones. 'Then

$$
\begin{aligned}
0=M_{1}[1] & \rightarrow[1]-X_{1}\left(X_{1}^{\prime} X_{1}\right)^{-1} X_{1}^{\prime}[1]=X_{1}\left(X_{1}^{\prime} X_{1}\right)^{-1} \Sigma X_{1 t} \\
& \Rightarrow[1]^{\prime}[1]=\Sigma X_{1 t}^{\prime}\left(\Sigma X_{1 t} X_{1 t}^{\prime}\right)^{-1} \Sigma X_{1 t} \Rightarrow 1=\left(T^{-1} \Sigma X_{1 t}^{\prime}\right)\left(T^{-1} \Sigma X_{1 t} X_{1 t}^{\prime}\right)^{-1}\left(T^{-1} \Sigma X_{1 t}\right)
\end{aligned}
$$

(ii) Write

$y_{t}=\mu_{0}+\mu t+\Theta_{i}, \quad \Delta y_{t}=\mu+\Delta \Theta_{i} ; \quad \Theta_{t} \equiv y_{t}-\mathbf{E} y_{t}$.

Since one of the columns of $X_{1}$ contains the constant term,

$$
\begin{aligned}
T^{-3 / 2}\left[y_{t-1}\right]^{\prime} M_{1}\left[\Delta y_{t}\right] \\
=T^{-3 / 2}\left(\left[\Theta_{t-1}\right]+[\mu t]\right)^{\prime} M_{1}\left[\Delta \Theta_{t}\right] \\
=T^{-3 / 2}\left[\Theta_{t-1}\right]^{\prime}\left[\Delta \Theta_{t}\right]-T^{-1 / 2}\left(T^{-1}\left[\Theta_{t-1}\right]^{\prime} X_{1}\right)\left(T^{-1} X_{1}^{\prime} X_{1}\right)^{-1}\left(T^{-1} X_{1}^{\prime}\left[\Delta \Theta_{t}\right]\right) \\
\\
\quad+T^{-3 / 2}[\mu t]^{\prime}\left[\Delta \Theta_{t}\right]-T^{-3 / 2}\left([\mu t]^{\prime} X_{1}\right)\left(X_{1}^{\prime} X_{1}\right)^{-1}\left(X_{1}^{\prime}\left[\Delta \Theta_{t}\right]\right) .
\end{aligned}
$$

Since $\Delta \Theta_{t}$ and $\Theta_{t}$ are stationary and ergodic, the first two of the four terms after the second equality in (6) converge in probability to zero. Apart from a factor of $\mu$, the third term is $T^{-3 / 2} \Sigma_{t} \Delta \Theta_{t}=$ $T^{-3 / 2}\left[-\Theta_{0}-\Theta_{1}-\cdots-\Theta_{T-1}+T \Theta_{T}\right]$. This has expectation zero. Since $y_{t}$ has an absolutely summable autocovariance function, it is easy to show that $\lim _{T \rightarrow \infty} \operatorname{var}\left\{T^{-3 / 2}\left[-\Theta_{0}-\Theta_{1}\right.\right.$ $\left.\left.-\cdots-\Theta_{T-1}+T \Theta_{T}\right]\right\}=0$. By Chebyschev's inequality, then, this term converges in probability to zero.

Let $C_{T}$ and $D_{T}$ be $k \times k$ diagonal matrices, $C_{T}$ having $T^{-2}$ in its $(1,1)$ element and $T^{-3 / 2}$ elsewhere on the diagonal, $D_{T}$ having $T^{-1 / 2}$ in its $(1,1)$ element and $T^{-1}$ elsewhere. Let $X_{1}^{*}$ be the $T \times k$ matrix with its $t$ th row

$$
\left(1 \Delta y_{t-1}-\mu \Delta y_{t-7}-\mu \ldots \Delta y_{t-k+1}-\mu\right) \equiv\left(1 \Delta \Theta_{t-1} \Delta \Theta_{t-}, \ldots \Delta \Theta_{t-k+1}\right) \equiv\left(1 Z_{t}^{\prime}\right) \equiv X_{1 t}^{* \prime} .
$$

Note that $X_{1}^{*}$ may be obtained from $X_{1}$ by a non-singular linear transformation. Apart from a factor of $\mu$, therefore, the fourth and final term after the second equality in (6) may be written

$$
[t]^{\prime} X_{1}^{*} C_{T}\left(T^{3 / 2} D_{T} X_{1}^{* \prime} X_{1}^{*} C_{T}\right)^{-1} D_{T} X_{1}^{* \prime}\left[\Delta \Theta_{t}\right]
$$

Now, $T^{-1 / 2} \Sigma \Delta \Theta_{t}=T^{-1 / 2}\left(-\Theta_{0}+\Theta_{T}\right) \stackrel{p}{\rightarrow} 0$ by Chebyschev's inequality, since $v_{t}$ has an absolutely summable autocovariance function. It was just shown that $T^{-3 / 2} \sum t \Delta \Theta_{t} \stackrel{p}{\rightarrow} 0$. It follows that

$[t]^{\prime} X_{1}^{*} C_{T} \stackrel{\mathrm{p}}{\rightarrow}(1 / 2,0 \ldots 0)$,

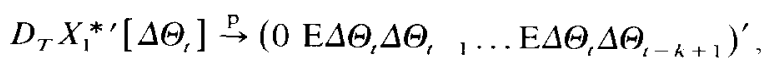

and that $T^{3 / 2} D_{T} X_{1}^{* \prime} C_{T}$ converges in probability to a block diagonal matrix with 1 in its $(1,1)$ position and $\mathrm{E} Z_{t} Z_{t}^{\prime}$ in its $(k-1) \times(k-1)$ lower right-hand block. This implies that $(8)$ converges in probability to zero. 
(iii) Write the square of the $t$-statistic for $\mathrm{H}_{0}: \gamma=1$ as

$\hat{\tau}^{2}=\left\{T^{3 / 2}(\hat{\gamma}-1)\right\}^{2} /\left\{\hat{\sigma}^{2}\left(T^{3}\left[y_{t-1}\right]^{\prime} M_{1}\left[y_{t-1}\right]\right)^{-1}\right\}$,

where $\hat{\sigma}^{2}$ is $T^{-1}$ times the sum of squared OLS residuals. It was just shown that $T^{3 / 2}(\hat{\gamma}-1)$ converges in probability to zero, $T^{-3}\left[y_{t-1}\right]^{\prime} M_{1}\left[y_{t-1}\right]$ to a non-zero constant. To establish that $\hat{\tau} \stackrel{p}{\rightarrow} 0$, then, it suffices to show that $\hat{\sigma}^{2}$ converges in probability to a non-zero constant.

Let $F_{T}$ be a $(k+1) \times(k+1)$ diagonal matrix with $T^{-3 / 2}$ in its $(k+1, k+1)$ position, $T^{-1 / 2}$ elsewhere on the diagonal; let $X$ be the $T \times(k+1)$ regression matrix; let $X^{*}$ be the $T \times(k+1)$ matrix with $\left(X_{1 t}^{* \prime} y_{t-1}\right) \equiv\left(1 Z_{t}^{\prime}, y_{t-1}\right)$ in its $t$ th row $\left[X_{1}^{*}\right.$ and $Z_{t}$ are defined in (8)]; let $M=I-$ $X\left(X^{\prime} X\right)^{-1} X^{\prime}=I-X^{*}\left(X^{* \prime} X^{*}\right)^{-1} X^{* \prime}$. Since $y_{t-1}=\mu_{0}+\mu(t-1)+\Theta_{t-1}, t=\mu^{-1}\left(y_{t-1}+\mu-\mu_{0}-\right.$ $\left.\Theta_{t-1}\right) \Rightarrow M[\delta t]=M\left[-\delta \mu^{-1} \Theta_{t-1}\right]$ since $y_{t-1}$ and a constant are among the columns of $X$. Define $\rho_{t}=e_{t}-\delta \mu^{-1} \Theta_{t-1}$. Then since $\hat{\sigma}^{2}=T^{-1}\left[\delta t+e_{t}\right]^{\prime} M\left[\delta t+e_{t}\right]$,

$\hat{\sigma}^{2}=T^{-1}\left[\rho_{t}\right]^{\prime}\left[\rho_{t}\right]-T^{-1}\left[\rho_{t}\right]^{\prime} X^{*} F_{T}\left(F_{T} X^{* \prime} X^{*} F_{T}\right)^{-1} F_{T} X^{* \prime}\left[\rho_{t}\right]$

Now, $T^{-1 / 2}\left\lfloor\rho_{t}\right\rfloor^{\prime} X^{*} F_{T} \stackrel{\mathrm{p}}{\rightarrow}\left(\mathrm{E} X_{1 t}^{* \prime} \rho_{t}(\mu / 2) \mathrm{E} \rho_{t}\right)=\left(\mathrm{E} X_{1 t}^{* \prime} \rho_{t} 0\right)$ by ergodicity, Lemma A.2 in West (1987), and the fact that $\mathrm{E} \rho_{t}=0$. By Lemmas 2.2 and A.2 in West $(1987), T^{-2} \Sigma y_{t-1} \mathrm{E} X_{1 t}^{*} \stackrel{\mathrm{P}}{\rightarrow}(\mu / 2) \mathrm{E} X_{1 t}^{*}$, $T^{-3} \Sigma y_{t-1}^{2} \stackrel{\mathrm{p}}{\rightarrow} \mu^{2} / 3$, and by ergodicity $T^{-1} \Sigma X_{1 t}^{*} X_{1 t}^{* \prime} \stackrel{\mathrm{p}}{\rightarrow} \mathrm{E} X_{1 t}^{*} X_{1 t}^{* \prime} ;$ this means that the $(k \times k)$ upper left-hand block of $\left(F_{\mathrm{T}} X^{* \prime} X^{*} F_{T}\right)^{-1}$ converges in probability to $\left\{\mathrm{E} X_{1 t}^{*} X_{1 t}^{* \prime}-(3 / 4) \mathrm{E} X_{1 t}^{*} \mathrm{E} X_{1 t}^{* \prime}\right\}^{-1}$. It follows that $\hat{\sigma}^{2} \stackrel{\mathrm{p}}{\rightarrow} \mathrm{E} \rho_{t}^{2}-\left(\mathrm{E} X_{1 t}^{*} \rho_{t}\right)^{\prime}\left\{\mathrm{E} X_{1 t}^{*} X_{1 t}^{* \prime}-(3 / 4) \mathrm{E} X_{1 t}^{*} \mathrm{E} X_{1 t}^{* \prime}\right\}^{-1} \mathrm{E} X_{1 t}^{*} \rho_{t}=\mathrm{E} \rho_{t}^{2}-$ $\left(\mathrm{E} Z_{t} \rho_{t}\right)^{\prime}\left(\mathrm{E} Z_{t} Z_{t}^{\prime}\right)^{-1} \mathrm{E} Z_{t} \rho_{t}$, which is non-zero since $\left(\rho_{t}, Z_{t}^{\prime}\right)^{\prime}$ has a variance-covariance matrix of full rank. The last equality follows since $\rho_{t}$ and $Z_{t}$ each have unconditional mean zero.

\section{References}

Bhargava, Alok, 1986, On the theory of testing for unit roots in observed time series, Review of Economic Studies LlII, 369-384.

Dickey, David A. and Wayne A. Fuller, 1979, Distribution of the estimators for autoregressive time series with a unit root, Journal of the American Statistical Association 74, 427-431.

Fuller, Wayne A., 1976, Introduction to statistical time series (Wiley, New York).

Kleidon, Allan W., 1986, Variance bounds tests and stock price valuation models, Journal of Political Economy 94, $953-1001$.

Mattey, Joe and Richard Meese, 1986, Empirical assessment of present value models, Econometric Reviews 5, 171-234.

Nelson, Charles R. and Charles I. Plosser, 1982, Trends versus random walks in macroeconomic time series: Some evidence and implications, Journal of Monetary Economics 10, 139-162.

Phillips, Peter C.B. and Pierre Perron, 1986. Testing for a unit root in time series regression, Cowles Foundation discussion paper no. 794 (Yale University, New Haven, CT).

Shiller, Robert J., 1981, Do stock prices move too much to be justified by subsequent changes in dividends?, American Economic Review 71, 421-436.

West, Kenneth D., 1987, Asymptotic normality, when regressors have a unit root, Manuscript (Princeton University, Princeton, NJ). 